\title{
Interdomain interactions within the two-component heme- based sensor DevS from Mycobacterium tuberculosis
}

E.T. Yukl, A. Ioanoviciu, P.R. Ortiz de Montellano, and P. Moënne-Loccoz

\section{Supporting information}

Figure S1. UV-vis spectra of ferric and ferrous GAF A DevS (red), GAF A/B DevS (blue), and FL DevS (black).

Figure S2. Low-frequency RR spectra of GAF A DevS- ${ }^{12} \mathrm{CO}$ (A, red), GAF A DevS- ${ }^{13} \mathrm{CO}$ (B, red), GAF A/B DevS $-{ }^{12} \mathrm{CO}$ (C, blue), GAF A/B DevS $-{ }^{13} \mathrm{CO}$ (D, blue), FL DevS $-{ }^{12} \mathrm{CO}$ (E, black), and FL DevS- ${ }^{13} \mathrm{CO}$ (F, black). ${ }^{12} \mathrm{CO}-{ }^{13} \mathrm{CO}$ difference spectra are also shown $(\lambda$ $\mathrm{exc}=413 \mathrm{~nm},<0.5 \mathrm{~mW}$ ).

Figure S3. $v(\mathrm{C}-\mathrm{O})$ versus $v(\mathrm{Fe}-\mathrm{CO})$ plots of heme protein- $\mathrm{CO}$ complexes. Data points for wt DevS constructs $(\mathrm{O})$ are compared with values for wt and distal mutants of myoglobin

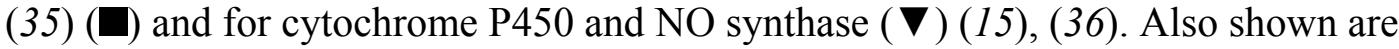
proximal histidine mutants of heme oxygenase $(\square)(37),(38)$ and GAF A DevS ( $\Delta$ ) (12).

Figure S4. Low-frequency RR spectra of ferrous nitrosyl adducts of GAF A DevS- ${ }^{14} \mathrm{NO}$ (A, red), GAF A/B DevS- ${ }^{14} \mathrm{NO}$ (B, blue), and FL DevS- ${ }^{14} \mathrm{NO}$ (C, black) illustrating frequencies of the $\delta(C C C)$ modes $\left(\lambda_{\text {exc }}=413 \mathrm{~nm}, 0.5 \mathrm{~mW}\right)$.

Figure S5. High-frequency RR spectra of ${ }^{14} \mathrm{~N}^{16} \mathrm{O}-{ }^{15} \mathrm{~N}^{16} \mathrm{O}$ difference spectra for the ferrous-nitrosyl adducts of GAF A DevS (Red), GAF A/B DevS (Blue), and FL DevS (Black) $\left(\lambda_{\text {exc }}=413 \mathrm{~nm}, 0.5 \mathrm{~mW}\right)$.

Figure S6. Low-frequency RR spectra of ${ }^{14} \mathrm{~N}^{16} \mathrm{O}$ and ${ }^{15} \mathrm{~N}^{16} \mathrm{O}$ adducts of FL DevS (Black) and ${ }^{14} \mathrm{~N}^{16} \mathrm{O}-{ }^{15} \mathrm{~N}^{16} \mathrm{O}$ difference spectra of GAF A DevS (Red), GAF A/B DevS (Blue), and FL DevS (Black) $\left(\lambda_{\text {exc }}=413 \mathrm{~nm}, 0.5 \mathrm{~mW}\right)$.

Figure S7. ${ }^{16} \mathrm{O}_{2}{ }^{18}{ }^{18} \mathrm{O}_{2}$ difference spectra of GAF A DevS and FL DevS oxygen adducts in $\mathrm{H}_{2} \mathrm{O}$ and $\mathrm{D}_{2} \mathrm{O}\left(\lambda_{\text {exc }}=413 \mathrm{~nm}, 1.0 \mathrm{~mW}\right)$. 

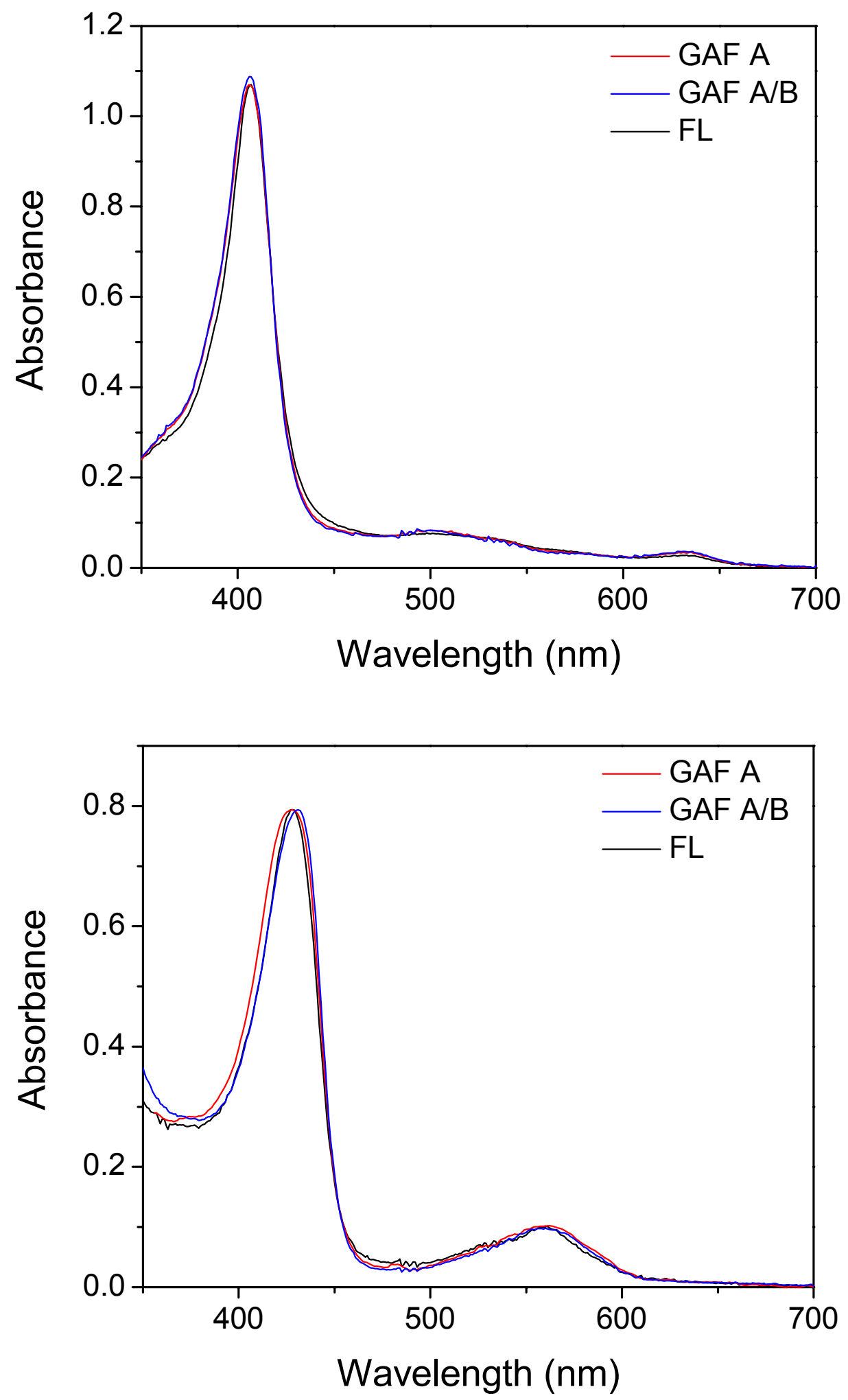

Figure S1 


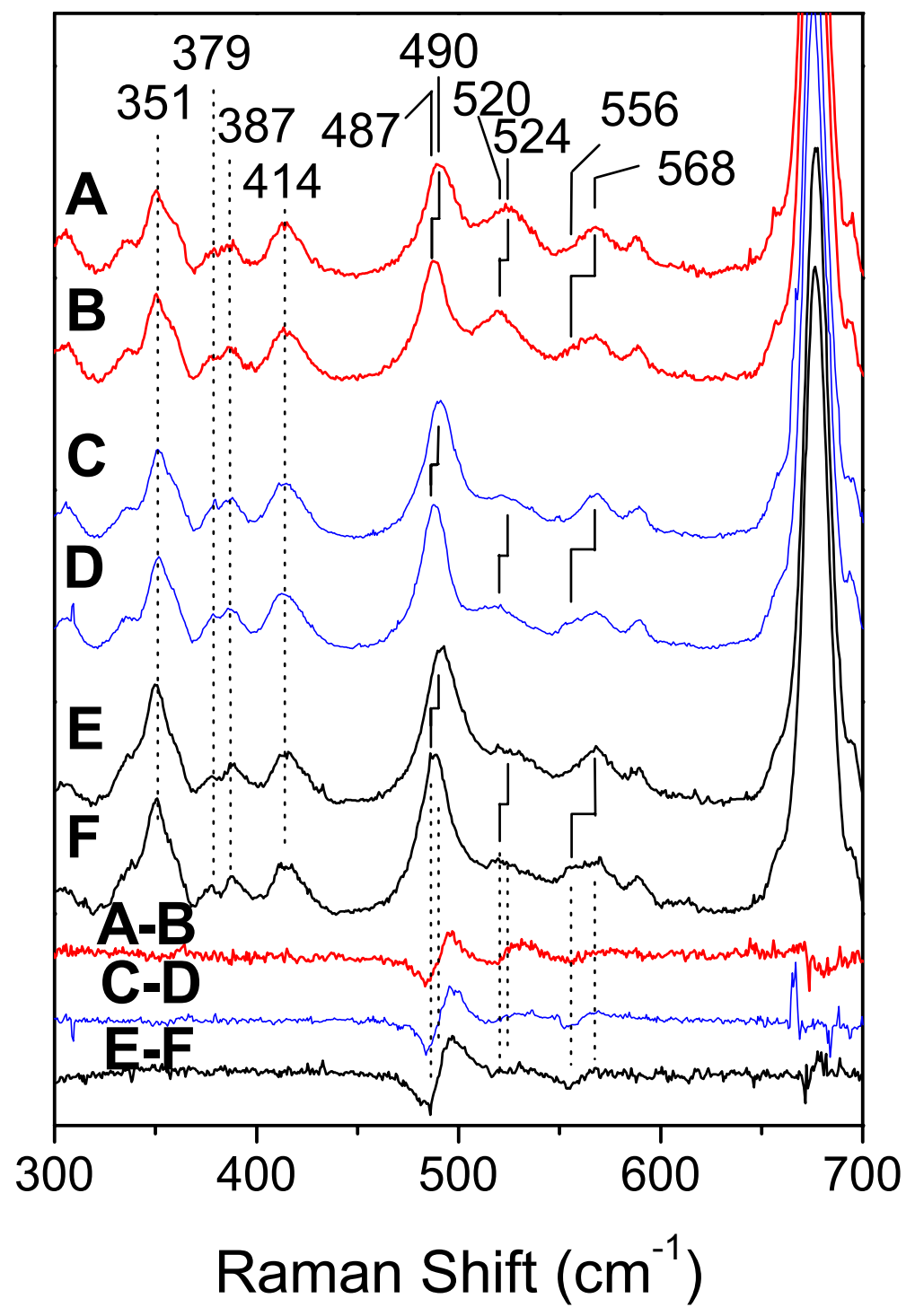

Figure S2 


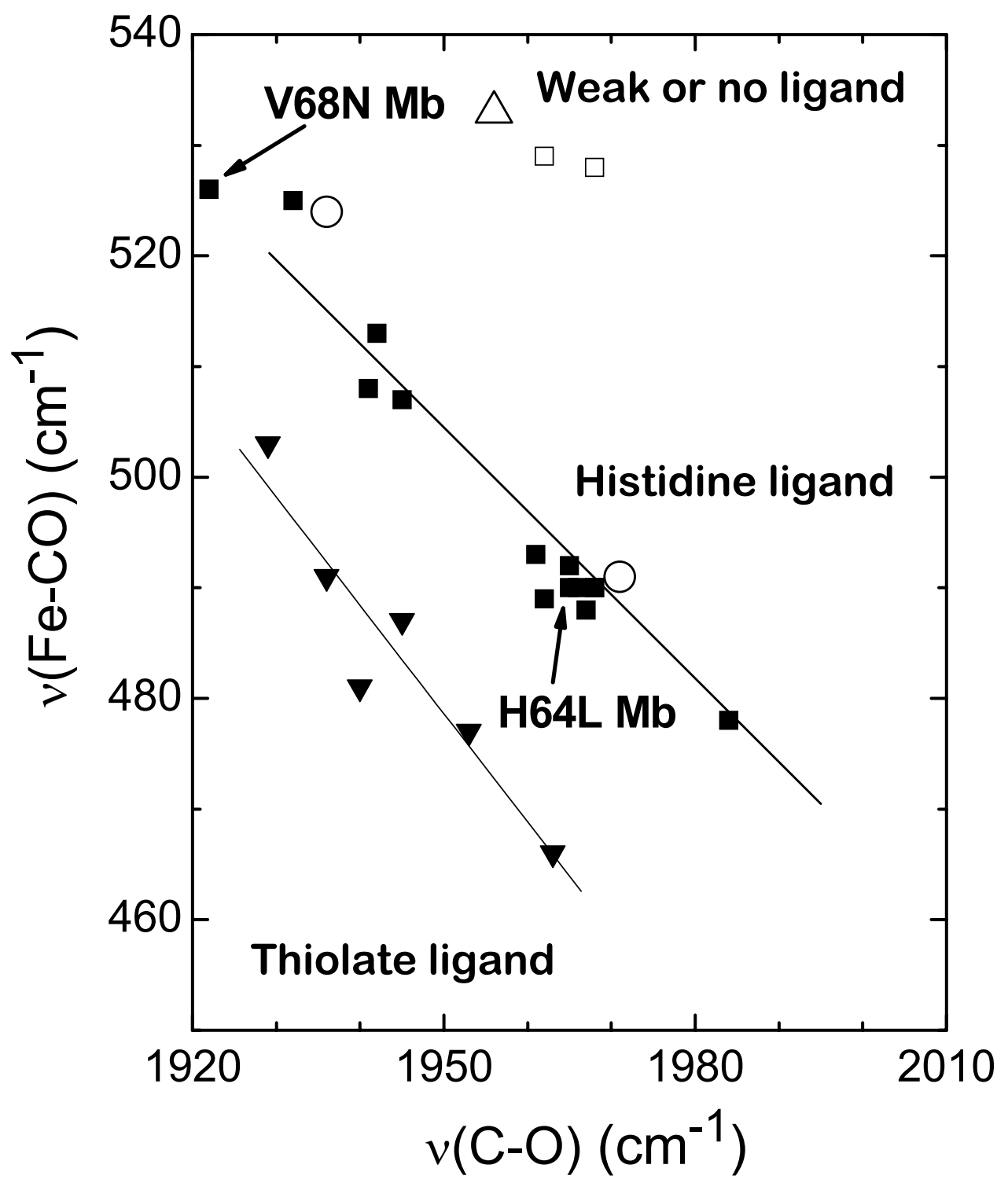

Figure S3 


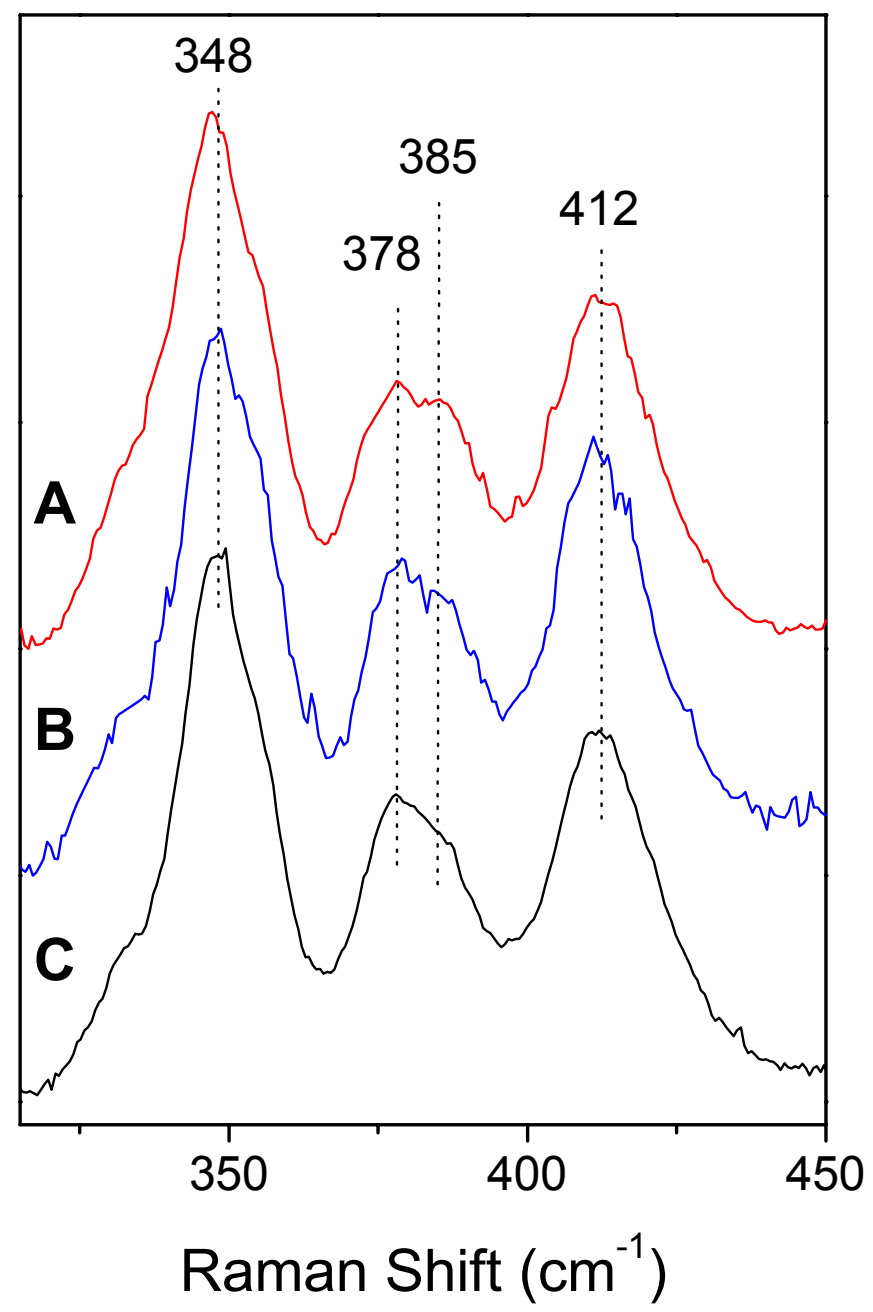

Figure S4 


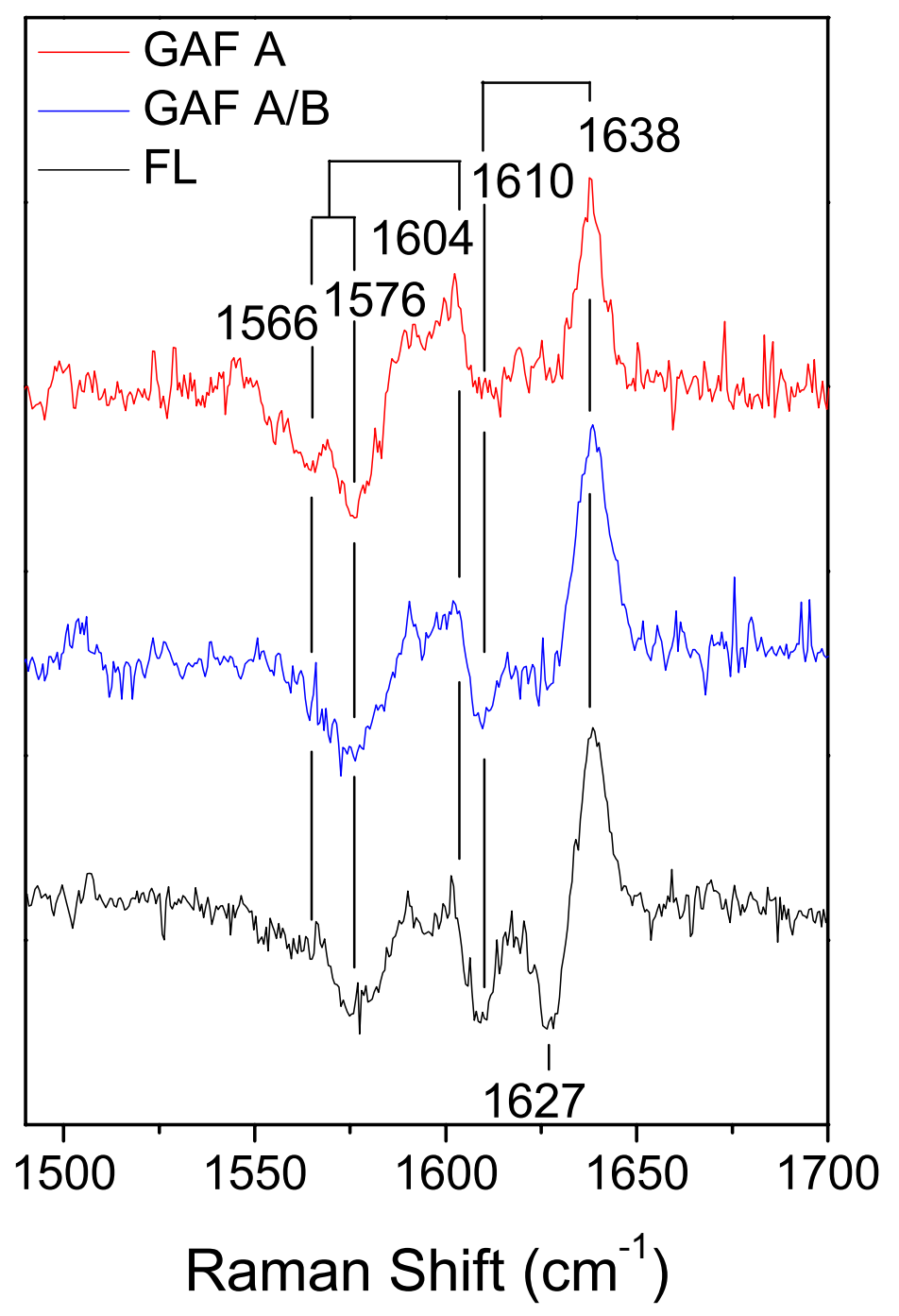

Figure S5 


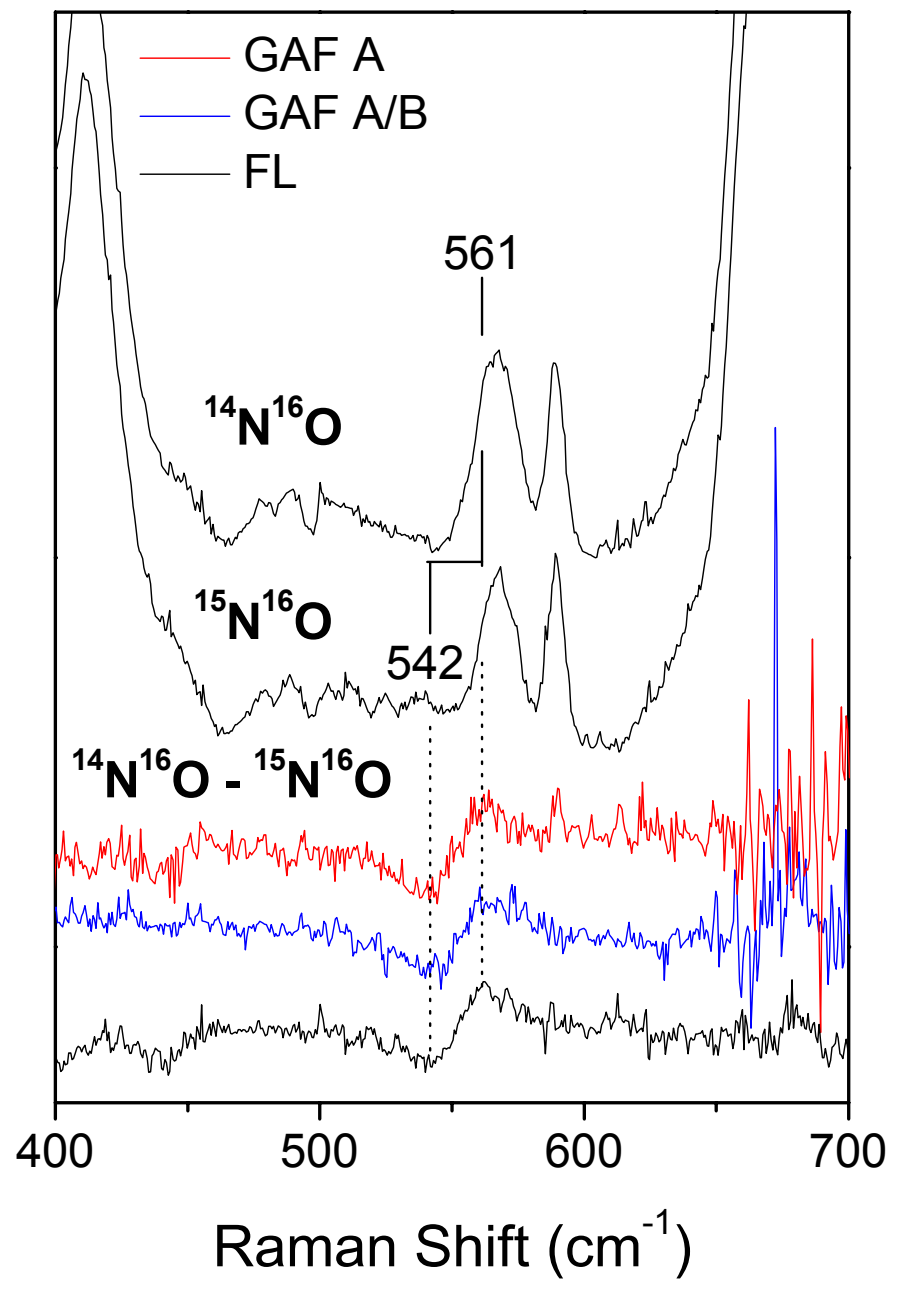

Figure S6 


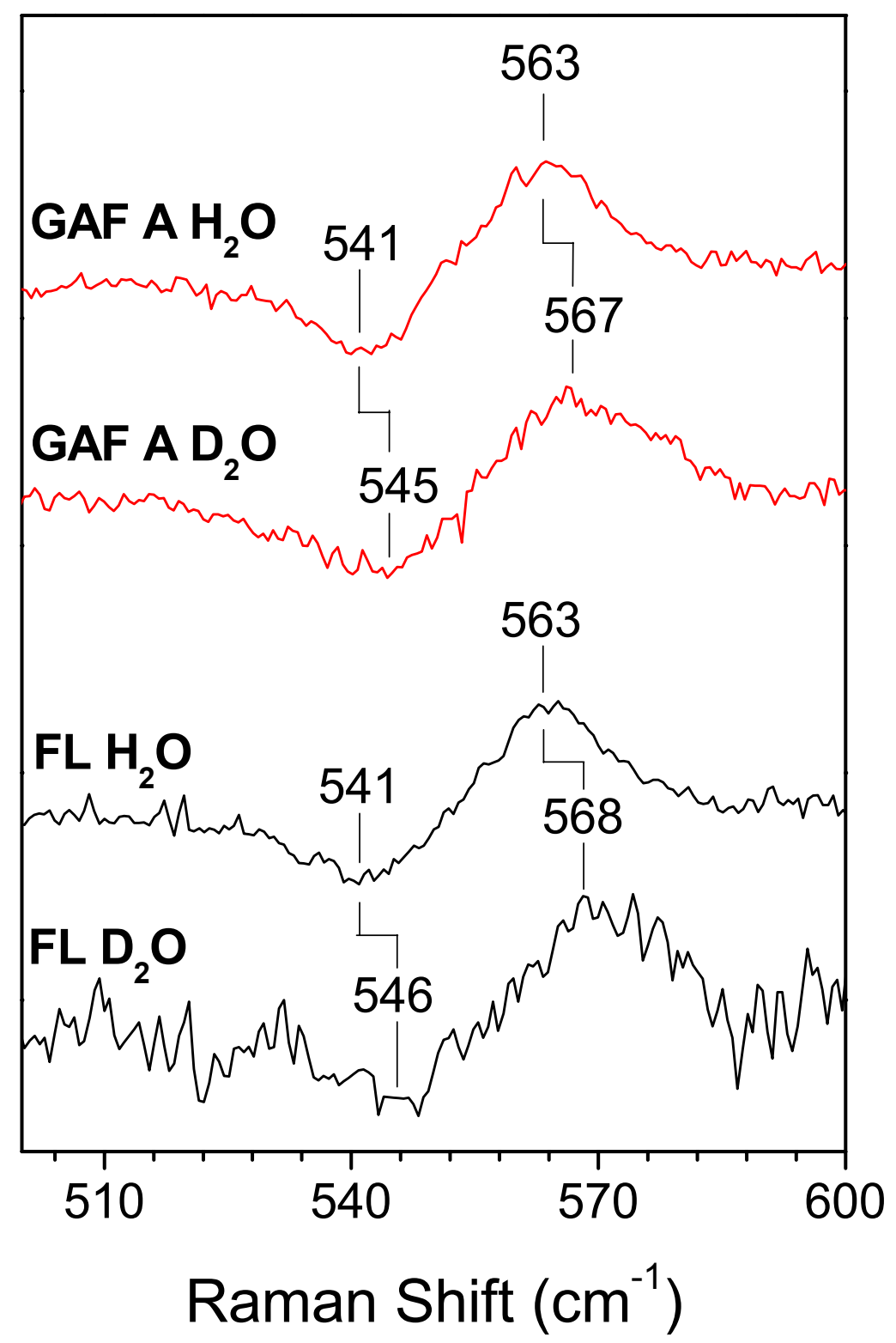

Figure S7 